whether by the ordinary process or by Mr. Collingwood's Method-and who has chanced to get a figure wrong at the very outset, which makes every subsequent figure wrong, has no warning of the fatal error till he has worked out the whole thing " to the bitter end," and has begun to test his Answer. Whereas, if working by my Method, he would have been warned of his mistake almost as soon as he made it, and would have been able to set it right before going any further.

As an aid to the Reader, I will give the Mental Process in full, for the and and 3 rd Columns of the first of the examples worked above.

The Divisor is 6997 (where $h=7, k=3$ ). Here you are supposed to have just entered the 28I in the Quotient. The Dividend, for these 2 Columns, is 1972 | 103 ; the Quotient is $28 \mathrm{r}$, and the Remainder 5946. The Test is hQ. $t^{2 n}+R$, (i.e. $\left.7 \times 281000+5946\right)$, the Mental Process being as follows. You write, on a separate slip of paper, the last 3 digits of $R$, viz. 946 , and carry the 5 into the next period, adding it to the $7 \times 28 \mathrm{I}$ : thus. " 5 and 7 , I2." Enter the 2, and carry the I. "I and 56, 57." Enter the 7, and carry the 5 . " 5 and I4, I9." Enter it. Having got your Test, try whether $(N+k Q)$ is equal to it. This you compute, comparing it with your Test, digit by digit, as you go on, thus. " 3 and 3,6 ." Observe it in the Test. "O and 24, 24." Observe the 4, and carry the 2. " 3 and 6 9." Observe it. "1972 and 0 , 1972." Observe it. The Test is satisfied.

For Divisors of the form $\left(t^{n} \pm k\right)$ there is no need to write out the Test : the numbers, which compose it, already occur in the working, and may be used as they stand.

Ch. Ch., Oxford, December 21, I897. Charles L. Dodgson.

\section{Optical Illusions produced by Observation of Rotating Spirals.}

IT is well known that if a spiral, such as represented in the figure, rotating in the opposite direction to the movement of the hands of a clock, be observed for some minutes, an impression of circles arising at the centre and disappearing at the periphery is produced; after protracted observation, on looking at printed matter or a person's face, the letters appear to move towards the centre while the face appears to become smaller and recede. If the spiral be rotated in the opposite direction, the circles

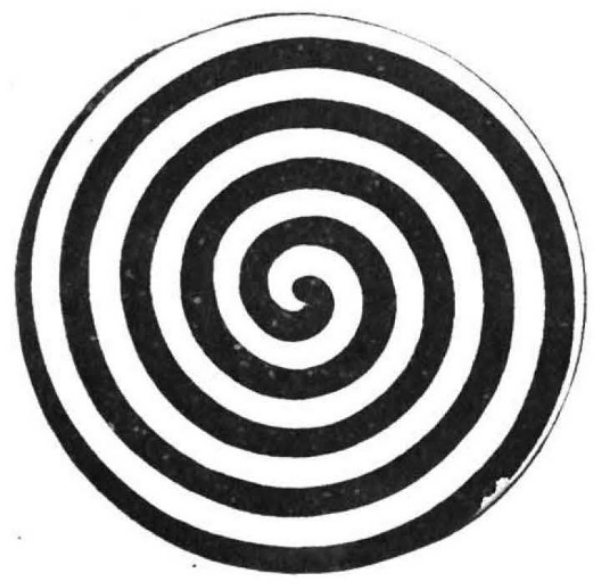

appear to be passing towards the centre, in which case the aftereffect is more marked and lasts longer, the letters naturally pass centrifugally while the face apparently increases in size.

The latter effect in my own observations has sometimes continued for fifty seconds, while the former never for more than twenty seconds. This effect is practically the same as the "waterfall phenomenon," in which case the banks appear to move upwards after gazing for some time at the falling water.
What I wish to draw attention to in this note is the effect which may be observed on closing the eyes after watching a well-illuminated rotating spiral.

If the direction of rotation has produced the impression of centripetally travelling rings, a star composed of radial lines consisting of small granular patches of light passing centrifugally will be noted for a second or two after closing the eyes. The granules pass centripetally if the direction of rotation be reversed. The star will appear symmetrical if the spiral be viewed normally, but if sideways the lines composing it will be curved and the star distorted: to some observers the centre of the star always appears to be further distant than the periphery. I have not been able to find any difference in time of duration of this after-image on rotating the spiral in the two directions.

The colour of the granules is always yellowish, whatever be the colour of the spiral and background. It is interesting to note that, as a rule, after-images of central production can be prophesied on psychological reasoning; while in the above case no one to my knowledge has been able to foretell the appearance of the star, and therefore I am inclined to think that the effect is retinal. O. F. F. Grünbaum.

\section{Poisonous Koda Millet.}

THERE have been several well-ascertained examples of poisoning from diseased or improperly-prepared Koda millet (Paspalum scrobiculatum) during the past year in India. Owing to the prevailing scarcity of the usual food-grains, it is probable that Koda millet has been extensively sold and eaten in localities where its use is ordinarily unknown.

I hoped to undertake an investigation myself into this matter, which is one of great importance both from the hygienic and economic points of view. After consultation, however, with Surgeon-Major van Geyzel, chemical examiner to the Government of Madras, $I$ have decided that any investigation worthy of the name would occupy far more time than I have to give to it. I therefore write to you with a view to eliciting the help of some worker in Britain or elsewhere who has the necessary leisure and facilities for work of this sort. I have samples of the grain, husked and unhusked, from different localities, and can supply the necessary references to the literature of the subject. The investigation, I take it, would have to be of a chemico-biological nature, and would be most appropriate, say, for a thesis, or in connection with some fellowship. I hope that some one who can carry the work right through, and will ascertain the exact source and constitution of the poison (? a volatile alkaloid), will write to me at the address given below. In another ten years, perhaps, there will be some attempt to provide the men and the facilities for such work, even in India, when the Victoria Institute is an accomplished fact.

Hygiene Laboratory, Medical College, Madras.

\section{Hermaphroditism in the Herring.}

I wish, with your permission, to record a singular "freak of nature" and, at the same time, to ask whether any similar observation has been made by others.

Some fresh herrings were served us here last evening for dinner, and among them was one which, on being opened, disclosed a roe, half of which was "hard" and the other half "soft." The ova and milt were respectively quite normal in appearance (and flavour), and melted imperceptibly one into the other about midway in the length of the roe, which was, itself, in an obviously natural and undisturbed condition.

Sea Lawn, Torquay, January 12.

Dan. Pidgeon.

\section{A Bright Meteor.}

ON Sunday night (December 12), at about 8.15, I witnessed the appearance of a magnificent meteor which seemed to travel from the south-east in the direction of Ursa Major. It broke up into a number of fiery balls of most brilliant colours. The atmosphere was clear and frosty and the moon very bright, but it was for the moment quite eclipsed by the brilliancy of this striking meteoric display. This may be the same phenomenon as that referred to in NATURE for January 6 (see page 228). I did not hear any sound like thunder at the time.

Susania Lehmann.

Wales Lodge, Wales, Sheffield, January i6. 